\title{
Aspectos destacados del Documento de consenso para el manejo de las arritmias asintomáticas
}

\author{
Dra. María Victoria Ramos
}

\section{Introducción}

Las arritmias asintomáticas son frecuentes en la práctica clínica. Aunque existe escasez de estudios que las aborden específicamente, muchas de ellas requieren celeridad en su diagnóstico y evaluación pronóstica para evitar consecuencias severas, como el accidente cerebrovascular (ACV) embólico, la insuficiencia cardíaca y la muerte súbita. Debido a esto, en febrero de 2019, la Asociación Europea del Ritmo Cardíaco (EHRA, por su sigla en inglés) publicó un documento de consenso con la evidencia disponible ${ }^{(1)}$, avalado por varias sociedades científicas del que comentaremos los aspectos más significativos.

El grado de percepción de los pacientes con anomalías del ritmo cardíaco es sumamente variable y el síntoma más frecuente son las palpitaciones, descritas como una sensación desagradable de pulsación o movimiento en el pecho o áreas adyacentes. Otros síntomas que pueden experimentarse son fatiga, disnea, disconfort torácico o síncope. Sin embargo, las arritmias cardíacas pueden presentarse de forma totalmente asintomática en algunos individuos, como ocurre frecuentemente en la fibrilación auricular (FA), taquicardias supraventriculares incesantes (TSV) y taquicardia ventricular no sostenida (TVNS), aun cuando su presencia influya en el pronóstico. La FA asintomática puede producir ACV, las arritmias ventriculares muerte súbita y todas las formas repetitivas y sostenidas de taquiarritmias pueden deteriorar la función ventricular izquierda.

Dado que la mayor parte de la evidencia se refiere a la evaluación y tratamiento de las arritmias sintomáticas, en la práctica diaria suele considerarse que la ausencia de síntomas implica un pronóstico más benigno y que habitualmente no requieren tratamiento.

\section{Arritmias y síntomas}

Así como algunos individuos pueden mantenerse completamente asintomáticos durante las arritmias, las palpitaciones pueden no corresponderse con una arritmia. No existen estudios que evalúen la relación entre síntomas y "carga" de arritmia. Múltiples factores pueden contribuir a que la arritmia sea sintomática o no, destacando el tipo y origen así como la presencia de cardiopatía estructural. Por ejemplo, las extrasístoles aisladas auriculares o ventriculares y salvas cortas probablemente generen menos síntomas que los episodios sostenidos.

El efecto hemodinámico de la arritmia está influido por la frecuencia cardíaca, el volumen sanguíneo circulante, la función ventricular izquierda y la presencia de comorbilidades. En el caso de las bradiarritmias, se considera que es necesaria una pausa sinusal o auriculoventricular mayor a $6-7$ segundos para causar síntomas. El uso de fármacos como betabloqueantes, calcioantagonistas y vasodilatadores puede afectar la habilidad del corazón para tolerar distintas arritmias.

\section{Extrasístoles y taquiarritmias auriculares no sostenidas}

La proporción de individuos con extrasístoles auriculares (EA) que experimentan síntomas es desconocida y tampoco existe evidencia de que el número de extrasístoles influya en su aparición. Actualmente se reconoce que la existencia de EA frecuentes y salvas pueden ser predictores independientes de taquicardia auricular y FA. Algunos estudios que evaluaron la actividad ectópica supraventricular excesiva (definida como EA >30/hora) mediante Holter de 48 horas observaron una asociación significativa con la presencia de ACV/muerte y admisiones por FA durante el seguimiento. Hasta el momento, no

Editora adjunta de la Revista Uruguaya de Cardiología.

Correspondencia: Dra. María Victoria Ramos. Correo electrónico: mariavictoriaramos@outlook.es

La autora declara no tener conflictos de intereses.

Recibido May 3, 2019; aceptado May 7, 2019. 
se sabe si el tratamiento con antiarrítmicos o la ablación en estos pacientes puede reducir el riesgo de desarrollar FA y sus consecuencias. Aunque no hay una definición clara de cuál es el número de EA que se considera excesivo, el documento de consenso propone como punto de corte $>500 / 24$ horas.

\section{Preexcitación ventricular asintomática}

La prevalencia del patrón de preexcitación ventricular (Wolff-Parkinson-White) en el electrocardiograma (ECG) se estima entre $0,1 \%$ y $0,3 \%$. El riesgo de muerte súbita en individuos sintomáticos es entre $3 \%$ y $4 \%$, por lo tanto se considera recomendación Clase I la realización de un estudio electrofisiológico (EEF) y la ablación de la vía accesoria. Sin embargo, en los casos asintomáticos, el riesgo oscila entre $0 \%$ y $0,6 \%$ en diferentes series.

La evaluación inicial del paciente con preexcitación ventricular asintomático podría incluir un test de ejercicio y Holter de 24 horas para valorar la presencia de bloqueo de la vía accesoria con frecuencias cardíacas incrementales o conducción intermitente. Ambas indican que el período refractario efectivo de la vía (PRE) es largo y el riesgo de muerte súbita muy bajo.

Aunque el valor predictivo positivo del EEF sería muy bajo para justificar su empleo de rutina, en una revisión sistemática acerca de la estratificación de riesgo arrítmico de eventos en pacientes con preexcitación asintomática, las Guías del Colegio Americano de Cardiología sobre TSV 2015 sugieren que puede ser beneficioso. Específicamente, este documento considera que los atletas profesionales o aquellos con ocupaciones de riesgo (pilotos, operadores de maquinaria) deberían realizarse un EEF. La preexcitación a joven edad, la inducción de taquicardia auriculoventricular durante el EEF, el PRE $<240 \mathrm{~ms}$ y la presencia de múltiples vías accesorias se han identificado como factores de riesgo que favorecen la ablación.

\section{Fibrilación y flutter auricular}

La FA asintomática hace referencia a aquella incidentalmente descubierta durante el examen clínico de rutina o detectada mediante screening y registrada por $\geq 30$ segundos en un ECG. Se considera que estos pacientes tienen una alta carga de arritmia, comparados con aquellos en los que es detectada a través de dispositivos implantados de monitoreo continuo.

La prevalencia real de FA asintomática es desconocida. Los reportes varían entre 10\%-40\%, según el perfil de riesgo de la cohorte evaluada, intensidad del monitoreo y seguimiento; la probabilidad de observarla aumenta progresivamente en los ancianos, en el sexo masculino y en aquellos con FA paroxística. Los pacientes sintomáticos pueden experimentar episodios de FA asintomática, especialmente luego de la ablación por catéter. Los datos disponibles actualmente sugieren que la FA asintomática implica peor pronóstico que la sintomática debido a una referencia más tardía para la estratificación del riesgo tromboembólico y tratamiento.

El manejo de los pacientes asintomáticos sigue los mismos principios que el de los sintomáticos. Un abordaje integrado como el ABC (basado en evitar el ACV con el uso de anticoagulación, controlar los síntomas mediante una estrategia de control del ritmo o de frecuencia, evaluar las comorbilidades cardiovasculares y factores de riesgo) resume los componentes centrales a considerar. Como el manejo de los síntomas no es relevante en estos casos, es importante intentar prevenir la perpetuación de la FA y disminuir el riesgo de cardiomiopatía inducida por taquicardia.

La prueba de control del ritmo con cardioversión eléctrica puede identificar individuos aparentemente asintomáticos que inconscientemente adaptan su estilo de vida o tienen síntomas atípicos. Los mismos principios de manejo se aplican a los pacientes con flutter auricular.

\section{Episodios de frecuencia auricular rápida}

Los episodios de frecuencia auricular rápida (AHREs, por su sigla en inglés), también denominados FA subclínica, se diferencian de la FA sintomática o asintomática por la forma en que son detectados: los AHREs se identifican solamente en aquellos individuos portadores de dispositivos implantados. La prevalencia reportada oscila entre $30 \%$ y $60 \%$ dado que la definición varía en los distintos estudios. La Sociedad Europea de Cardiología en su Guía sobre el diagnóstico y tratamiento de la FA de 2016, considera AHREs a los episodios de más de 5-6 minutos de duración y con frecuencia $>180$ cpm detectados por el dispositivo(2). La evidencia disponible no demostró relación temporal entre los episodios de AHREs y la ocurrencia de ACV, poniendo en discusión si son la causa o simples marcadores de riesgo de miocardiopatía auricular. AHREs prolongados ( $>24$ horas) se asocian con mayor riesgo de ACV isquémico o embolismo sistémico. Sin embargo, aún no hay estudios que indiquen si la anticoagulación es beneficiosa en el caso de AHREs de corta duración. 


\section{Extrasístoles ventriculares}

La presencia de extrasístoles ventriculares (EV) aisladas es normal en la mayoría de los individuos, incluyendo jóvenes sanos. Habitualmente se generan por actividad focal y menos frecuentemente por mecanismos de microrreentrada. Las EV frecuentes pueden ser marcadoras de un sustrato cardíaco anormal ya sea eléctrico, isquémico o estructural. En estos casos, las EV pueden originarse por automatismo aumentado, actividad desencadenada o reentrada. Características específicas de las EV como alta carga, complejidad (duplas, tripletas o salvas), origen multifocal e incremento de su frecuencia con el ejercicio pueden asociarse con resultados adversos.

No existe un punto de corte absoluto del número de EV necesario para considerar la presencia de enfermedad subyacente, pero se ha observado que en atletas aparentemente sanos con $>2.000 \mathrm{EV}$ en 24 horas el riesgo de hallar una patología fue $30 \%$. Aun en ausencia de cardiopatía demostrada, la presencia de EV de moderada a alta incidencia constituye un marcador de mortalidad cardiovascular por todas las causas. La morfología de las EV provee información adicional en el diagnóstico, ya que algunos sitios de ectopía se reconocen como benignos, especialmente en los tractos de salida ventriculares. Las más frecuentes se originan en el tracto de salida ventricular derecho (morfología de bloqueo completo de rama izquierda en la derivación V1 con transición entre V3-V4), aunque se ha visto que en algunas oportunidades pueden ser la expresión atípica de una cardiopatía arritmogénica del ventrículo derecho. En estos casos, la ausencia de alteraciones en el ecocardiograma y la resonancia magnética cardíaca permite descartar la patología. Infrecuentemente, EV consideradas como "benignas" pueden desencadenar taquicardia ventricular polimórfica o fibrilación ventricular debido a un intervalo corto de acoplamiento (fenómeno de R-sobre-T), como se observa en el contexto de isquemia, alteraciones electrolíticas, síndrome de QT largo o repolarización precoz; pero también puede ocurrir en corazones "normales".

Las EV frecuentes (definidas usualmente como $>10 \%-15 \%$ del total de latidos en 24 horas) pueden deteriorar la función del ventrículo izquierdo (cardiomiopatía inducida por EV), proceso que potencialmente revierte mediante tratamiento antiarrítmico o ablación por catéter. No todos los pacientes con EV frecuentes desarrollan cardiomiopatía, mientras que en otros aparece con cargas menores $(<10 \%)$. Los factores asociados con disfunción ventricular incluyen: complejos ectópicos con mayor duración del QRS, EV originadas en el epicardio, activación retrógrada auricular y EV interpoladas.
$\mathrm{El}$ documento sugiere que la presencia de EV $>500 / 24$ horas es motivo suficiente para evaluar una eventual enfermedad subyacente, mientras que en atletas se considera un punto de corte $>2.000 / 24$ horas. Si los estudios solicitados son normales, en los individuos asintomáticos no se requiere tratamiento, pero se recomienda el control anual de la función ventricular en los que posean EV $>10 \%$. En los pacientes sintomáticos o que desarrollan disfunción ventricular se recomienda tratamiento médico o ablación por catéter.

\section{Taquicardia ventricular}

La definición de TVNS implica tres o más latidos ventriculares consecutivos a $>100 \mathrm{cpm}$ con duración $<30$ segundos. Su prevalencia aumenta con la edad (0,7\%-10\%) y es muy común en la cardiopatía isquémica (30\%-80\%). Mientras que la TVNS puede ser asintomática, los episodios sostenidos frecuentemente generan síntomas.

Dentro de las arritmias ventriculares, dos entidades requieren mención específica: la taquicardia ventricular bidireccional y la torcida de puntas. La primera puede ser asintomática en el síndrome de Andersen-Tawil, siendo otras causas la intoxicación digitálica y la taquicardia ventricular polimórfica catecolaminérgica. La torcida de puntas es un tipo de taquicardia ventricular polimórfica que se observa exclusivamente en los casos de QT largo, congénito o adquirido. La evaluación de estos casos tiene por objetivo identificar una enfermedad cardíaca subyacente, específicamente la cardiopatía isquémica, mediante la realización de ECG de 12 derivaciones, ecocardiograma, Holter, estudios de laboratorio y prueba de estrés (según la situación clínica). En una segunda línea de evaluación podrían considerarse: el estudio invasivo y no invasivo de las arterias coronarias, la resonancia magnética cardíaca, el EEF, los tests farmacológicos (ajmalina y flecainida) para desenmascarar un eventual síndrome de Brugada y los estudios genéticos.

El pronóstico se considera usualmente benigno en los que no se demuestra patología subyacente, aunque la TVNS polimórfica requiere evaluación detallada y tratamiento que puede incluir fármacos, ablación y hasta el implante de un cardiodesfibrilador.

En pacientes con cardiopatía estructural, la presencia de TVNS es un signo ominoso. En este contexto únicamente los betabloqueantes han demostrado disminuir la mortalidad. El tratamiento médico óptimo con inhibidores de la enzima conversora de angiotensina y antagonistas de los receptores de mineralocorticoides es el primer paso en quienes 
Tabla 1. Principales recomendaciones del Documento de consenso para el manejo de las arritmias asintomáticas .

\begin{tabular}{ll}
\hline Extrasístoles auriculares & Recomendación \\
& $\begin{array}{l}\text { Realizar búsqueda de cardiopatía estructural y modificación de los factores de } \\
\text { riesgo cardiovascular. }\end{array}$ \\
& $\begin{array}{l}\text { La asociación de EA }>500 / 24 \text { h con FA de corta duración puede favorecer el } \\
\text { inicio de anticoagulación. }\end{array}$ \\
& $\begin{array}{l}\text { EA de baja o moderada incidencia sin FA documentada no es indicación de } \\
\text { anticoagulación. }\end{array}$ \\
& $\begin{array}{l}\text { El seguimiento clínico es razonable en pacientes asintomáticos de bajo riesgo } \\
\text { (preexcitación intermitente o EEF sin factores de riesgo). }\end{array}$ \\
& La ablación por catéter se recomienda en pacientes de alto riesgo (PRE \\
& $<240$ ms, FA preexcitada o taquicardia auriculoventricular inducible o \\
& múltiples vías accesorias).
\end{tabular}

La ablación por catéter debe considerarse en atletas profesionales o en aquellas personas con ocupaciones de riesgo.

Fibrilación auricular

Los pacientes con FA asintomática deben ser anticoagulados de acuerdo con su riesgo de $\mathrm{ACV}$ igual que los sintomáticos.

El control de frecuencia con fármacos debe realizarse en pacientes con FA y

elevada respuesta ventricular para disminuir el riesgo de cardiomiopatía inducida por taquicardia.

Episodios de frecuencia auricular rápida

Los pacientes con AHREs deben considerarse con mayor riesgo embólico.

Debe realizarse una evaluación detallada para detectar la presencia de FA.

La anticoagulación puede considerarse en casos con score $\mathrm{CHA}_{2} \mathrm{DS}_{2}-\mathrm{VASc} \geq 2$.

Extrasístoles ventriculares

AHREs per se no requieren tratamiento antiarrítmico.
$\mathrm{EV}>500 / 24$ h deben evaluarse para descartar la presencia de enfermedad
cardíaca estructural, isquémica o eléctrica subyacente.
Deben tratarse las EV en aquellos pacientes con sospecha de cardiomiopatía
inducida por taquicardia.

EV muy frecuentes (>20\%) son marcadores de mortalidad cardiovascular por todas las causas y requieren un seguimiento intensivo.

Taquicardia ventricular no sostenida

Los pacientes con TVNS asintomática deben evaluarse detalladamente para
descartar la presencia de cardiopatía estructural, isquémica o eléctrica.
La TVNS en pacientes asintomáticos con función ventricular izquierda $\geq 40 \%$
usualmente no requiere tratamiento antiarrítmico específico.
La cardiomiopatía inducida por taquicardia es un diagnóstico de exclusión.
El manejo incluye tratamiento de la insuficiencia cardíaca, control de
frecuencia en el caso de FA (cuando no sea posible control de ritmo) y control de
ritmo en el resto de los casos.

Cardiomiopatía inducida por taquicardia

Se prefiere la ablación por catéter para control de ritmo en arritmias auriculares y ventriculares persistentes.

Bradiarritmias

En individuos con síncope, la presencia de bradiarritmia asintomática severa o de pausas $>6$ s debe considerarse diagnóstica.

La bradicardia sinusal asintomática no requiere tratamiento

En verde: existe evidencia de que el procedimiento o tratamiento es beneficioso y efectivo; en amarillo: acuerdo general o evidencia científica de la utilidad/eficacia del procedimiento o tratamiento; en rojo: no se recomienda realizar el procedimiento o tratamiento. EA: extrasístoles auriculares; FA: fibrilación auricular; PRE: período refractario efectivo; ACV: accidente cerebrovascular; AHREs: episodios de frecuencia auricular rápida; EV: extrasístoles ventriculares; TVNS: taquicardia ventricular no sostenida; s: segundos. 
presentan disfunción del ventrículo izquierdo. Luego de descartar la existencia de enfermedad arterial coronaria, el implante de un cardiodesfibrilador está recomendado en sujetos con fracción de eyección del ventrículo izquierdo $<35 \%$ y TV sostenida.

La ocurrencia de TV en atletas, aun cuando sea asintomática, obliga a descartar cardiopatía estructural (con especial atención en descartar la taquicardia ventricular polimórfica catecolaminérgica) o el uso de sustancias ilegales. El pronóstico es bueno en ausencia de enfermedad subyacente y la interrupción de la actividad física puede resolver los casos de TV inducida por ejercicio, aunque algunos requieren ablación por episodios persistentes.

Mientras que las EV son comunes durante el embarazo, la TV es excepcional y si se descarta cardiopatía estructural, puede prescindirse de tratamiento específico. En niños con arritmias asintomáticas y corazón normal no se ha observado beneficio del tratamiento antiarrítmico.

\section{Bradicardia asintomática}

Pocos estudios han examinado el valor pronóstico de la bradicardia asintomática en la población general. Los bloqueos auriculoventriculares de primer y segundo grado Mobitz I, son frecuentes en individuos jóvenes en reposo y en atletas.

Las pausas $>3$ segundos son poco frecuentes, usualmente no generan síntomas y su presencia no implica peor pronóstico o la necesidad de implante de marcapasos, dado que la pérdida de conciencia se produce luego de pausas de 6-7 segundos, como se refirió previamente. Los episodios nocturnos de bradicardia (bradicardia sinusal o bloqueo auriculoventricular) deben orientar a pesquisar la existencia de apnea obstructiva del sueño, ya que su tra- tamiento con presión positiva continua en la vía aérea las previene en 80\%-90\%. Las pausas de 3-5 segundos son frecuentes en los pacientes con FA y no se requiere tratamiento en ausencia de síntomas.

En el caso de bloqueo auriculoventricular es crucial distinguir la localización nodal de la infranodal, ya que en la última se requiere implante de marcapasos. Los bloqueos auriculoventriculares de segundo grado Mobitz II y de tercer grado deben tratarse con marcapasos aun en ausencia de síntomas, debido a la potencial severidad.

La tabla 1 muestra las principales recomendaciones del documento de consenso.

María Victoria Ramos,

https://orcid.org/0000-0002-6349-2781

\section{Bibliografía}

1. Arnar D, Mairesse G, Boriani G, Calkins H, Chin A, Coats A, et al. Management of asymptomatic arrhythmias: a European Heart Rhytm Association (EHRA) consensus document, endorsed by the Heart Failure Association (HFA), Heart Rhytm Society (HRS), Asia Pacific Heart Rhytm Society (APHRS), Cardiac Arrhythmia Society of Southern Africa (CASSA), and Latin America Hearth Rhytm Society (LAHRS). Europace 2019. doi: 10.1093/europace/euz046 Epub ahead of print

2. Kirchhof $\mathbf{P}$, Benussi S, Kotecha $\mathbf{D}$, Ahlsson A, Atar D, Casadei B, et al. Guía ESC 2016 sobre el diagnóstico y tratamiento de la fibrilación auricular, desarrollada en colaboración con la EAGTS. Ver Esp Cardiol. 2017 Ene [consulta 2019 May 5];70(1): [84p.]. doi: 0.1016/j.recesp.2016. 11.014. Fe de erratas en: Rev Esp Cardiol.2017; 70(11):1031. 